\title{
House-dust mite allergy: mapping of Dermatophagoides pteronyssinus allergens for dogs by two-dimensional immunoblotting
}

\author{
Luís Miguel Lourenço Martins ${ }^{1,2}$, Andreia Grilo Marques ${ }^{1,3}$, Luísa Maria Dotti Silva Pereira ${ }^{2,4}$, Ana Goicoa ${ }^{5}$, \\ Saul José Semião-Santos ${ }^{6}$, Ofélia Pereira Bento ${ }^{2,7}$
}

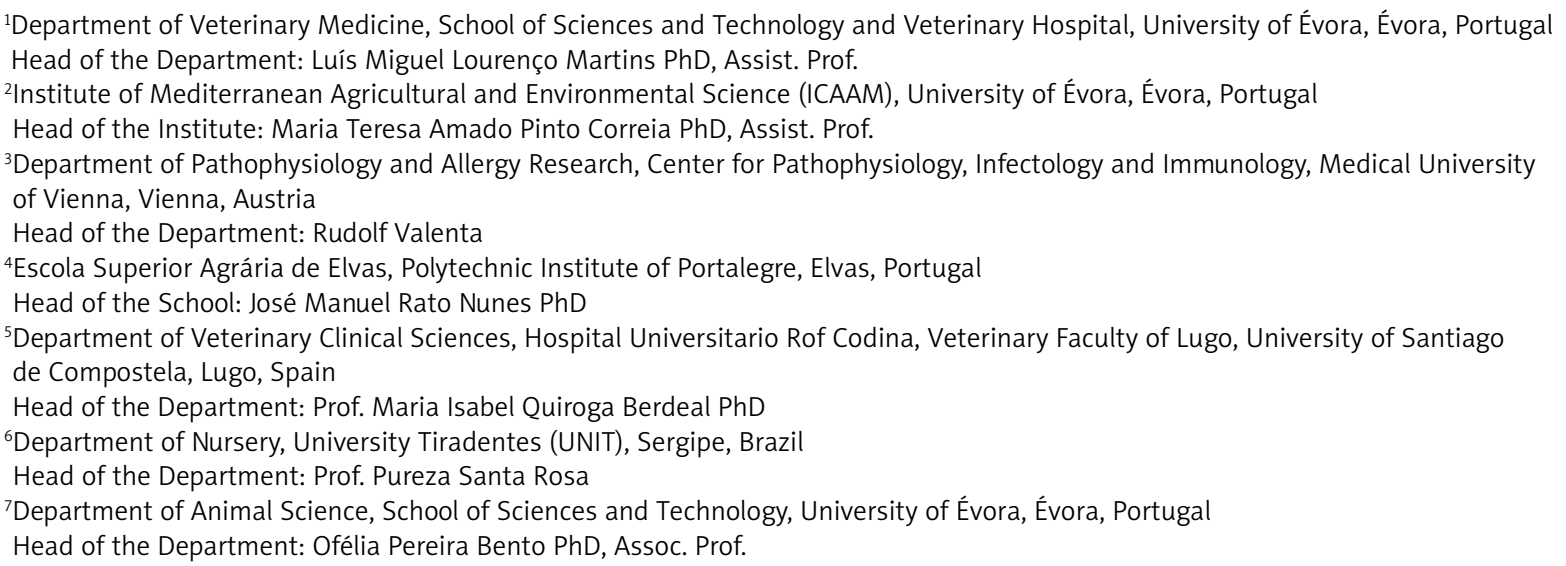

Postep Derm Alergol 2015; XXXII, 2: 73-81

DOI: $10.5114 / p d i a .2015 .48067$

\begin{abstract}
Introduction: Specific immunotherapy has shown to be very useful for allergy control in dogs, with a common success rate ranging from $65 \%$ to $70 \%$. However, this efficacy could probably be improved and the identification of individual allergomes, with the choice of more adequate molecular allergen pools for specific immunotherapy, being the strategy.

Aim: To map Dermatophagoides pteronyssinus (Der p) allergens for mite-sensitized atopic dogs, for better understanding how individual allergograms may influence the response to house-dust mite immunotherapy.

Material and methods: To identify the Der $p$ mite allergome for dogs, 20 individuals allergic to dust-mites and sensitized to Der $p$, were selected. The extract from Der $p$ was submitted to isoelectric focusing (IEF), one-dimensional (1-D) and two-dimensional (2-D) sodium dodecyl sulphate polyacrylamide gel electrophoresis (SDS-PAGE). Separated proteins were blotted onto polyvinylidene difluoride (PVDF) membranes and immunoblottings were performed with patient sera. Allergen-bound specific IgE was detected.

Results: Eleven allergens were identified from isoelectric focusing (IEF), as well as from 1-D SDS PAGE. From 2-D SDS-PAGE, 24 spots were identified.

Conclusions: Several similarities were found between dog and human allergograms and no absolute correlation between sensitization and allergy was observed either. As in humans, different individual allergograms do not seem to implicate different clinical patterns, but may influence the response to specific immunotherapy. The molecular epidemiology approach in veterinary allergy management, by the characterization of individual patients' allergoms and by choosing the best molecular allergen pool for each patient could also improve the efficacy of allergy immunotherapy.
\end{abstract}

Key words: allergens, atopy, dog, house-dust mite, immunoblotting.

Address for correspondence: Luís Miguel Lourenço Martins, Department of Veterinary Medicine, School of Sciences and Technology and Veterinary Hospital, University of Évora, Núcleo da Mitra, 7000-093 Évora, Portugal, phone: +351 919498570, fax: +351 266760944, e-mail: ImIm@uevora.pt

Received: 26.08.2014, accepted: 12.11.2014. 


\section{Introduction}

Allergen-specific immunotherapy, especially for aeroallergens, has proven to be useful for allergy control, promoting substantial clinical improvement in 65-75\% of the cases [1] and should be prescribed as directed as possible [2, 3]. Nevertheless, those figures may be safely improved by using more accurate allergen pools for specific immunotherapy, which is already being studied for human allergy management [4-8].

Concerning mite allergens, the molecular epidemiology concept may also lead to important innovations since the knowledge of mite allergen structure may allow a better interpretation of cross reactions between allergens from different mite species. It may also lead us to a further explanatory molecular diagnosis and, therefore, to a more effective immunotherapy, based on patient IgE-recognized allergens [4]. Masuda et al. [9] had already performed several Immunodot and IgE-enzyme-linked immunosorbent assay (ELISA) inhibition studies, using Dermatophagoides pteronyssinus (Der $p$ ) and D. farinae (Der $\mathrm{f}$ ) extracts and major allergens (Der $\mathrm{p} 1$, Der $\mathrm{p} 2$, Der $f 1$ and Der $f$ 2), demonstrating important evidence of cross-reactivity between the two species and the usefulness of those methods for the diagnosis of IgE-mediated diseases in dogs. Regarding D. pteronyssinus, Asturias et al. [5] engineered two hybrid molecules (QM1 and QM2) derived from the two major allergens, Der $p 1$ and Der $p 2$, with less IgE-binding activity but preserved immunogenicity. This strategy fulfilled the basic requirements of hypoallergenic molecules and showed suitability for the future specific immunotherapy of mite-allergic patients.

Recent studies performed by Kubota et al. [10] with Cryptomeria japonica pollen allergens, using sera from sensitized dogs with atopic dermatitis showed important differences in terms of individual allergen recognition between humans and dogs. In fact, a molecule that has shown to be a major allergen for humans may be a minor one for dogs and vice versa. Sometimes, an allergen for a given species may not be so for another. More recently, Ognjenovic et al. [11] reported several similarities and differences between dog and human sensitization to the Ambrosia artemisiifolia pollen, with $81 \%$ recognition of the $38 \mathrm{kDa}$ band, belonging to the Amb a 1 group, between sensitized dogs.

For a precise allergy diagnosis, or indication of allergen-directed eviction measures [12], as well as to improve the efficacy of allergen-specific immunotherapy [13], further fine tune laboratory information on the molecular diagnosis seems to be truly helpful. Other very important studies in this context have been performed, either characterizing major or minor Dermatophagoides allergens for dogs with atopic dermatitis [14] or even characterizing and cloning $D$. farinae allergens like Der f 15 [15]. Another study by Raffan et al. [16] focusing on the prevalence of the Dermatophagoides group
1 allergens (Der p 1 and Der f 1 ) in homes with no dogs, healthy dogs and Dermatophagoides-sensitized atopic dogs, concluded that sensitization to D. farinae would result mainly from cross-reaction, because $D$. pteronyssinus was rather prevalent, while $D$. farinae was found to be rare.

Following very useful concepts, such as component-resolved diagnosis and immunotherapy (CRD and CRIT) [17-19], while protein microarray systems are not available for veterinary proposals, characterization of patient serum IgE spectrotypes (individual allergograms) by immunoblotting techniques, with electrophoretic-separated molecular allergens, may provide relevant information, especially for immunotherapy decision [13].

\section{Aim}

In this work, the authors intended to further map $D$. pteronyssinus allergens for mite-sensitized atopic dogs, in order to start understanding how individual allergograms may influence the response to house-dust mite immunotherapy.

\section{Material and methods}

\section{Patient selection and characterization}

At dermatology and allergy outpatient consultations, carried out at the University of Évora (Évora, Portugal) and at the RofCodina (Lugo, Spain) Veterinary Hospitals, all the patients were submitted to an extensive query for anamnestic and clinical parameters, in which they were selected by filling the clinical criteria defined by the International Task Force of Canine Atopic Dermatitis for atopy diagnosis [20]. For further selection, patients were submitted to intradermal testing (IDT) with D. pteronyssinus, D. farinae, Lepidoglyphus destructor, Acarus siro and Tyroglyphus putrescentiae extracts, and positive (histamine solution) and negative (solvent extract) controls from Bial Aristegui (Bilbao, Spain). Specific IgE for a panel of the most common mites and pollens was also determined according to Lee et al. [21] (Univet, Barcelona, Spain). Twenty of those, mostly indoor patients and presenting atopic dermatitis with perennial clinical manifestations, which tested positive in the IDT and presented with specific IgE for D. pteronyssinus, were selected. Only 1 patient (no. 2) was already undergoing mite-specific immunotherapy.

\section{Allergen extract preparation}

Lyophilized D. pteronyssinus mites (Allergon, Angelholm, Sweden) were submitted to extraction by $2 \mathrm{~h}$ orbital stirring at $1: 10$ in $4^{\circ} \mathrm{C}$ double-distilled water, for IEF, and in phosphate buffered saline (PBS) for SDS-PAGE. The extract was centrifuged at $3500 \mathrm{~g}$ for $10 \mathrm{~min}$ at $4^{\circ} \mathrm{C}$ and supernatant protein concentration measured ac- 
cording to the Warburg Christian Method [22] in a spectrophotometer (Beckman DU530 UV-VIS, Beckman Coulter, Brea, USA).

\section{Protein separation}

\section{IEF}

The D. pteronyssinus extract (protein concentration of $7.5 \mathrm{mg} / \mathrm{ml}$ ) was subjected to IEF in $5 \%$ polyacrylamide gel (Acrylamide/Bisacrylamide from Bio-Rad, Hercules, USA) with a 12 : 1 mix of ampholytes from $\mathrm{pH} 4-6.5$ and pH 3-10 (GE Healthcare, USA). Separated proteins were then blotted onto methanol-activated 0.2- $\mu \mathrm{m}$-pore PVDF membranes (Bio-Rad) according to the technique described by Martins et al. [23] for isoelectric-focused protein transference. The extract was also subjected to IEF, to produce 1-D gel strips for the 2-D gel electrophoresis.

\section{SDS-PAGE}

The D. pteronyssinus extract was treated with SDS, under non-reducing conditions, and submitted to $12 \%$ PAGE. Separated proteins were blotted under electrotransference [24] onto methanol-activated 0.2- $\mu$ m-pore PVDF membranes in a Trans-Blot Semi-Dry Transfer Cell (Bio-Rad). Two-dimensional separation was performed by $12 \%$ SDS-PAGE of the 1-D IEF strips and separated proteins were also blotted onto methanol-activated 0.2- $\mu \mathrm{m}$ pore PVDF membranes.

\section{Antigen detection - immunoblotting}

One-dimensional blotted membranes were cut in 3-mm-wide strips, immersed for $2 \times 0.5 \mathrm{~h}$ in blocking buffer (1\% non-fat dry milk - 0.1\% Tween-20 in Tris-buffered saline, at $\mathrm{pH}$ 7.4) at room temperature and each strip was incubated overnight with individual patient sera, diluted at $1: 10$ in $1 \mathrm{ml}$ blocking buffer. Eight patients (sera no. 1-6, 9 and 10) were tested with strips from IEF and 20 (sera no. 1-20) with SDS-PAGE ones. One-dimensional immunoblots from IEF were also performed with 2 pools of 4 sera, each (P1: sera no. 1, 3, 5 and 6, and P2: sera no. 2, 3,9 and 10), allowing to obtain spectrotypes as complex as possible, as those individuals, together, recognized allergens through a wide $\mathrm{pH}$ range. For sera no. 2, two samples were used: 2B (before immunotherapy) and 2A (after 1-year house-dust mite immunotherapy). After incubation, strips were washed for $4 \times 5 \mathrm{~min}$ in $0.9 \% \mathrm{NaCl}$ $-0.1 \%$ Tween-20. For specific IgE detection, strips were incubated for $2 \mathrm{~h}$ with horseradish peroxidase-conjugated goat anti-dog-IgE antibody (Bethyl Laboratories, Inc., Montgomery, USA) at $1: 1000$ in blocking buffer, followed by $4 \times 5 \mathrm{~min}$ washes in $0.9 \% \mathrm{NaCl}-0.1 \%$ Tween-20. Another $4 \times 5$ min washing cycle was carried out and specific IgE visualization performed by chromogenic reaction with 3.3'-diaminobenzidine/ $\mathrm{NiCl}_{2}$ (Sigma-Aldrich, St. Louis, USA) in Tris-buffered saline at $\mathrm{pH}$ 7.4.
Two-dimensional immunoblottings were performed by incubating blotting membranes with a pool of 15 patient sera, selected for their representative IgE-binding patterns in IEF or SDS-PAGE, diluted to 1 : 10 in $18 \mathrm{ml}$ blocking buffer. Specific IgE was detected likewise with 1-D strips.

Three controls were performed with 1-D strips: C1 without serum; C2 - without serum or anti-dog IgE and C3 - with a pool of sera from 4 non-atopic dogs, negative for specific IgE to house-dust mites. Control for 2-D immunoblotting was performed with sera from 4 non-atopic dogs, negative for specific IgE to house-dust mites.

To compare specificity of pattern recognition between secondary antibodies, 1-D IEF-separated strips were also submitted to immunoblotting with a four-patient pool of sera (no. 1, 3, 5 and 6) and incubated with mouse anti-dog-lgE biotin-labeled monoclonal antibody (AbDSerotec, Kidlington, UK) at $1: 500$ in blocking buffer, followed by $4 \times 5$ min washes in $0.9 \% \mathrm{NaCl}-0.1 \%$ Tween-20 and $1 \mathrm{~h}$ incubation with ExtrAvidin-alkaline phosphatase (Sigma-Aldrich) at $1: 5000$. Another $4 \times 5$ min washing cycle was done and specific IgE visualization was performed by chromogenic reaction with nitrobluetetrazolium/5-bromo-4-chloro-3-indolyl phosphate (Sigma-Aldrich) in substrate buffer. Control was performed: C4 - with sera from 4 non-atopic dogs, negative for specific IgE to house-dust mites.

\section{Results}

All patients presented with specific IgE and positive IDT, at least to D. pteronyssinus and D. farinae. Mean specific IgE to D. pteronyssinus was 357.8 ELISA absorbance units (EAU) (min. of 35 and max. of 967) with a standard deviation (SD) of 277.06. Mean IDT score was 1.8 (min. of 1 and max. of 3 ) in a range from 1 to 4 with a SD of 0.68 .

From IEF separations, at least 11 allergens were identified from pl 5.4 to 6.8 (Figure 1 and Table 1). The mean number of recognized IEF allergen bands/patient was 6.25 (min. of 3 and max. of 9) with a SD of 2.86. Although, other IgE-binding proteins, probably including several isoallergens, were identified in P1 and P2 strips (Figure 1). From 1-D SDS-PAGE, 11 allergen bands were also identified by patient sera. The mean number of recognized PAGE allergen bands/patient was 5.33 (min. of 3 and max. of 8) with a SD of 1.55. Six allergen bands, with molecular weights (MW) close to 13.5, 23.5, 32.5, 47.5, 70 and 210-220 kDa showed major recognition (Figure 2 and Table 2). The majority of patients presented complex spectrotypes (Figures 1 and 2). Two-dimensional separation disclosed multiple allergen spots from $\mathrm{pl}<4.6$ to $>6.9$ and from 13.5 to 210-220 kDa (Figure 3 A). A diagram of the 2-D identified allergome is shown in Figure $3 \mathrm{~B}$, for a clearer representation. Controls, either using or not anti-dog lgE secondary antibodies, C1 and C2, respectively, did not show band recognition in blots. Controls with sera from non-atopic 


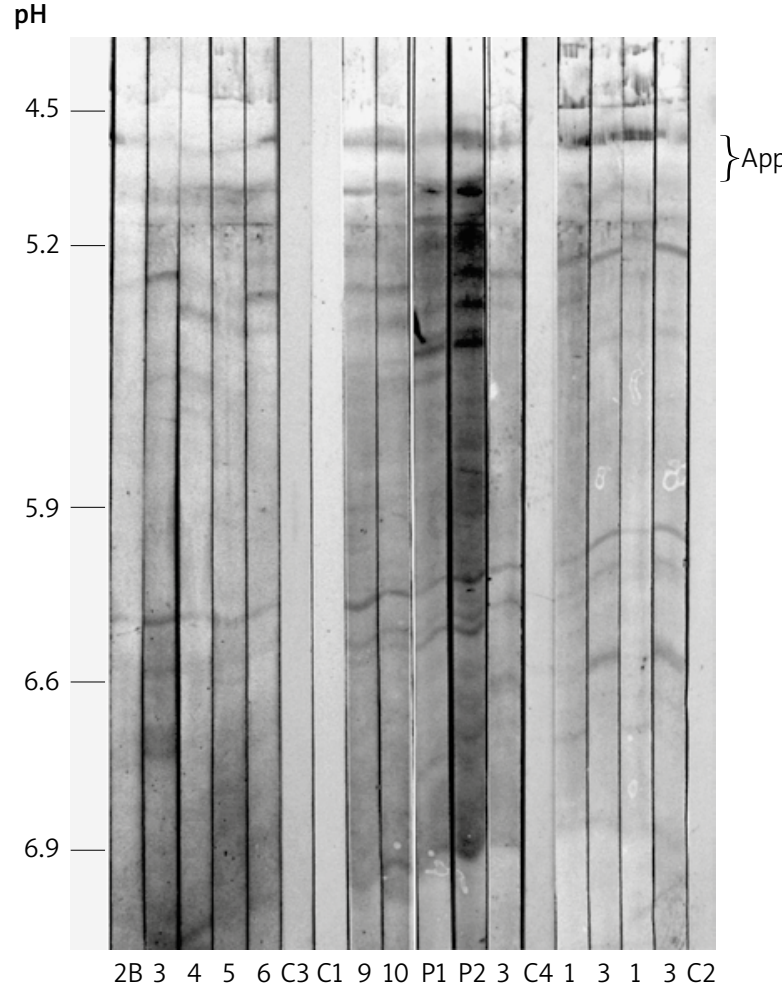

$C$ - Controls, $P$ - pools of sera, revealed with biotin-labeled mouse anti-dog IgE, App - sample application line.

Figure 1. Patient IgE immunoblots (patients/lanes no. 1-6, 9 and 10) from IEF of Der p extract, revealed with horseradish peroxidase-conjugated goat anti-dog-lgE

patients, negative for specific lgE to house-dust mites (C3 and (4) did not show recognition, either. No significant difference in specificity was found between the two anti-dog IgE systems tested. Patient no. 2, which has undergone 1 year of mite-specific immunotherapy, increased the complexity of the $D$. pteronyssinus spectrotype by the additional recognition of a $28.5 \mathrm{kDa}$ band (Figure 2 - Lanes $2 \mathrm{~B}$ and $2 \mathrm{~A}$, and Table 2), despite a clear clinical improvement.
A significant positive correlation $(r=0.843)$ was verified between specific IgE to D. pteronyssinus and respective IDT scores, with a marked variability in individual specific IgE levels to D. pteronysinus $(S D=277.06)$ and a relatively low variation in individual IDT scores (SD = 0.68). No significant correlation was found between specific IgE levels and the number of IEF allergen bands ( $r=$ 0.189), and between IDT scores and the number of IEF allergens $(r=0.246)$. A moderate positive correlation was observed between specific lgE levels and the number of SDS PAGE allergens ( $r=0.407)$, and between IDT scores and the number of SDS PAGE allergens $(r=0.431)$, while a negative one $(r=-0.430)$ was found between the number of IEF and SDS PAGE allergens.

\section{Discussion}

No clear clinical differences were recognized among patients. Hence no specific IgE level, IDT score or pattern of spectrotype was possible to relate to a specific clinical pattern. Nevertheless, IDT scores seemed to be a reliable parameter in terms of correspondence to the level of specific IgE to D. pteronyssinus. However, such high correlation may not occur between specific IgE levels or IDT scores and the number of allergens observed from SDS PAGE.

Several similarities were found between dog and human D. pteronyssinus spectrotypes. Like humans [23], dogs also recognized pl 5.4, 5.5-5.7, 5.8-5.9 and 6.1-6.2, and 21.5-24.5, 28.5-29, 37, 58, 108.5-110 and 208-210 $\mathrm{kDa}$ allergen bands. Another study performed by Le Mao et al. [25] with sera from mite-sensitized humans established several important features about Der f allergens, associated to a close cross-reactive pattern with Der $\mathrm{p}$, which was also confirmed by Masuda et al. [9]. In fact, the 24-25 kDa Der f1, with 10 isoforms from pl 5.9-8.2, may correspond to Der 1 , represented here by the 23.5$27 \mathrm{kDa} a, b, n, p$ and $q$ spots, with $\mathrm{pl}<4.6-6.6$ (Figure $3 \mathrm{~B}$ ). Der f2, with 14-15 kDa and 3 isoforms (pl 7.0, 7.2 and 7.4) recognized by a monoclonal antibody against Der $\mathrm{p} 2$,

Table 1. Allergens recognized from Der $p$ IEF

\begin{tabular}{|c|c|c|c|c|c|c|c|c|c|c|c|}
\hline \multirow[t]{2}{*}{ Patients } & \multicolumn{11}{|c|}{ Allergens (estimated $\mathrm{pl}^{*}$ ) } \\
\hline & 5.4 & 5.5 & 5.6 & 5.7 & 5.9 & 6.2 & 6.3 & 6.5 & 6.6 & 6.7 & 6.8 \\
\hline 1 & $X$ & $X$ & $X$ & & & & $X$ & $x$ & $X$ & & $x$ \\
\hline $2 \mathrm{~B}$ & $x$ & & & & & & $X$ & $x$ & & & \\
\hline 3 & $x$ & & $x$ & $x$ & $x$ & & $x$ & $x$ & $x$ & $x$ & \\
\hline 4 & & $X$ & $X$ & $X$ & & $X$ & $X$ & $x$ & $X$ & & \\
\hline 5 & $x$ & $x$ & $x$ & & & $x$ & $x$ & & $x$ & & $x$ \\
\hline 6 & $x$ & $X$ & $X$ & & & & $X$ & $x$ & $X$ & & \\
\hline 9 & $x$ & $x$ & $x$ & $x$ & $x$ & & $x$ & $x$ & $x$ & $x$ & \\
\hline 10 & $x$ & $x$ & $x$ & $X$ & $x$ & & $X$ & $X$ & $X$ & $X$ & \\
\hline
\end{tabular}

*Isoelectric point. 


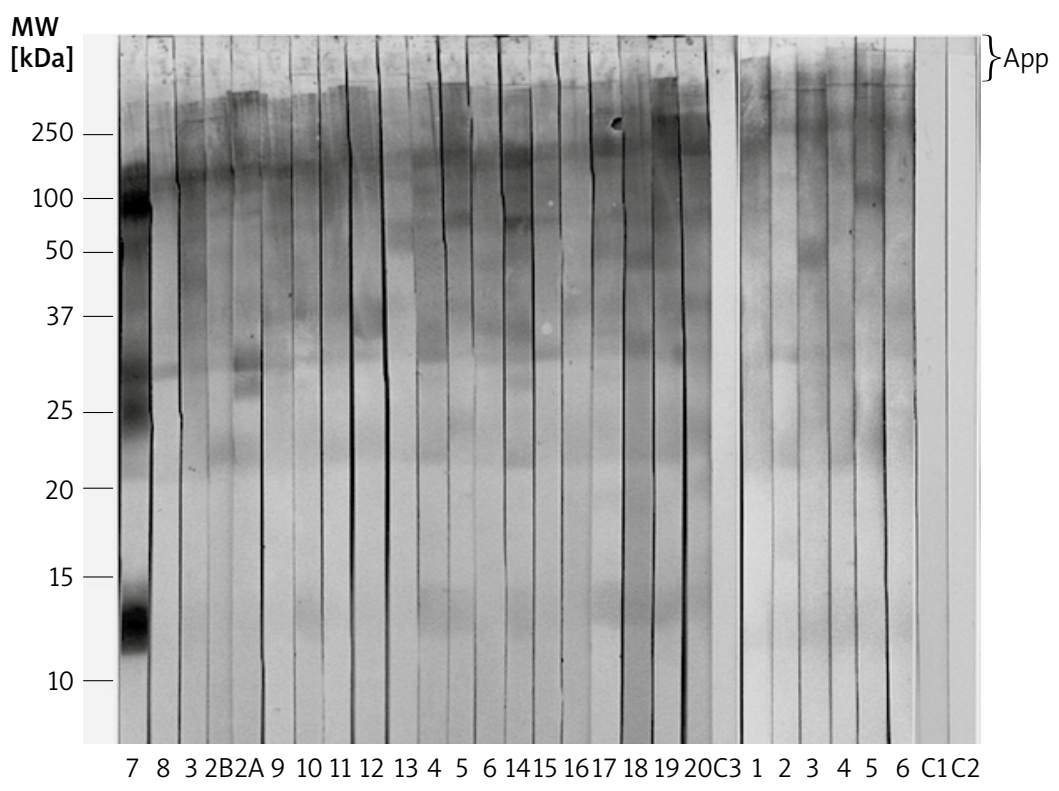

$C$ - Controls, $P$ - pools of sera, App - sample application line.

Figure 2. Patient IgE immunoblots (patients/lanes no. 1-20) from SDS-PAGE of Der p extract, revealed with horseradish peroxidase-conjugated goat anti-dog-lgE

Table 2. Allergens recognized from Der $p$ SDS-PAGE 1-D

\begin{tabular}{|c|c|c|c|c|c|c|c|c|c|c|c|}
\hline \multirow[t]{2}{*}{ Patients } & \multicolumn{11}{|c|}{ Allergens (estimated $\mathrm{MW}^{\star}[\mathrm{kDa}]$ ) } \\
\hline & 13.5 & 23.5 & 28.5 & 32.5 & 35.5 & 37 & 47.5 & 58 & 70 & 110 & $210-220$ \\
\hline 1 & $x$ & $x$ & & $x$ & & $x$ & $x$ & & $x$ & & $x$ \\
\hline $2 B$ & & $x$ & & $x$ & & & $x$ & & $x$ & $x$ & $x$ \\
\hline $2 \mathrm{~A}$ & & $x$ & $x$ & $x$ & & & $x$ & & $x$ & $x$ & $x$ \\
\hline 3 & $x$ & $x$ & & $x$ & & $x$ & $x$ & & $x$ & & $x$ \\
\hline 4 & $x$ & $x$ & & $x$ & & & & $x$ & $x$ & $x$ & $x$ \\
\hline 5 & $x$ & $x$ & & $x$ & $x$ & & & & $x$ & & $x$ \\
\hline 6 & & & & $x$ & $x$ & $x$ & $x$ & & & & $x$ \\
\hline 7 & $x$ & $x$ & $x$ & $x$ & & & $x$ & & $x$ & $x$ & $x$ \\
\hline 8 & & $x$ & & $x$ & & & & & & & $x$ \\
\hline 9 & & $x$ & & $x$ & & & $x$ & & $x$ & & $x$ \\
\hline 10 & $x$ & & & $x$ & & & $x$ & & $x$ & & $x$ \\
\hline 11 & & $x$ & & $x$ & & & & & & & $x$ \\
\hline 12 & & & & $x$ & $x$ & & & & & & $x$ \\
\hline 13 & & & & & & & $x$ & $x$ & $x$ & $x$ & $x$ \\
\hline 14 & $x$ & $x$ & & $x$ & $x$ & & & & $x$ & & $x$ \\
\hline 15 & & & & $X$ & & & & & $x$ & & $X$ \\
\hline 16 & & $x$ & & & $x$ & & & & & & $x$ \\
\hline 17 & $x$ & $x$ & & $x$ & $x$ & & $x$ & & $x$ & & $x$ \\
\hline 18 & $x$ & $x$ & & $x$ & & & $x$ & & & & $x$ \\
\hline 19 & $x$ & $x$ & & $x$ & & & $x$ & & $x$ & & $x$ \\
\hline 20 & & $x$ & & $x$ & $x$ & & & & $x$ & & $x$ \\
\hline
\end{tabular}

${ }^{*}$ Molecular weight. 


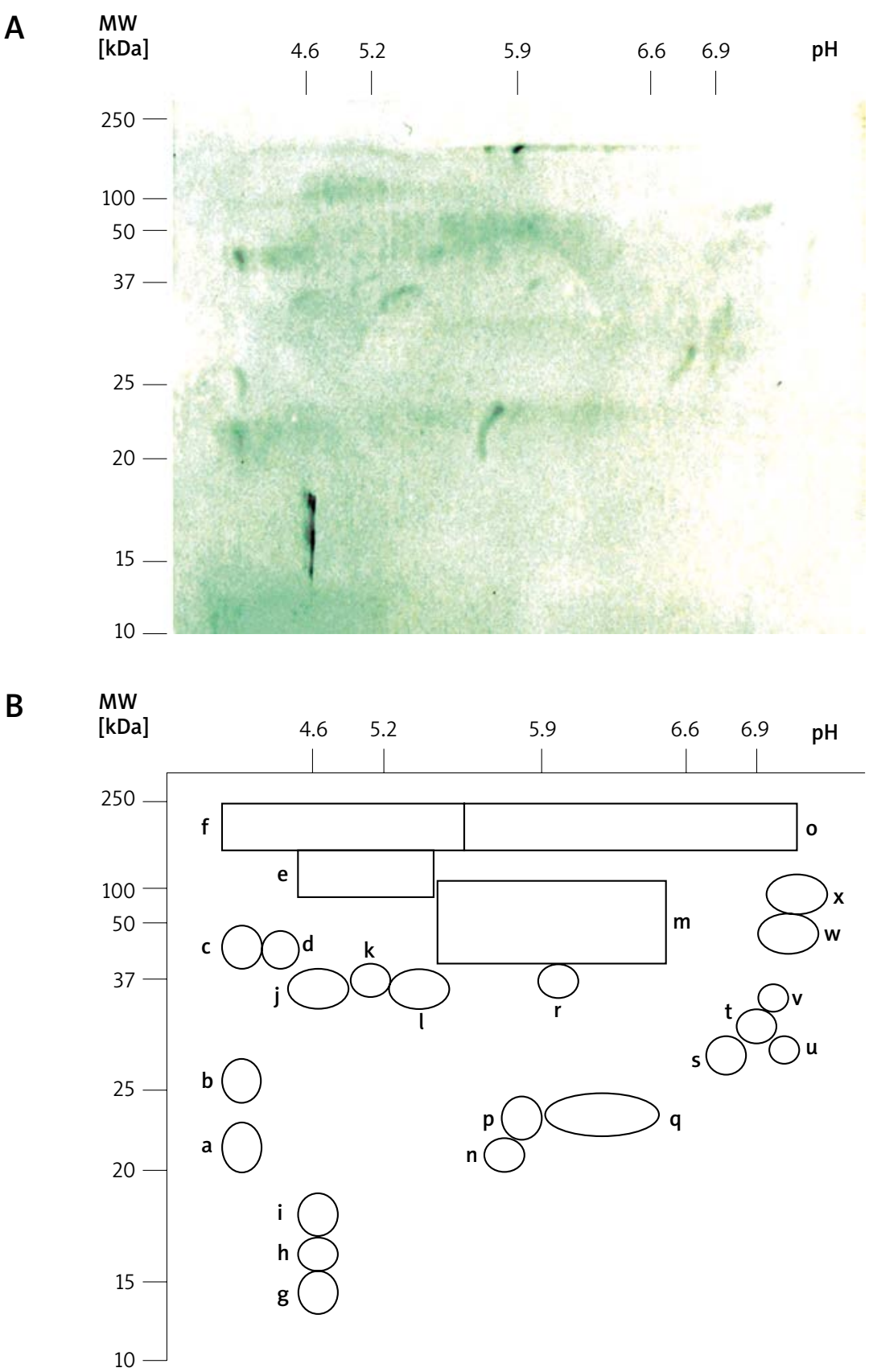

Figure 3. A - IgE immunoblot with a pool of sera from 2-D Der $p$ separation (dog Der $p$ allergogram), revealed with horseradish peroxidase-conjugated goat anti-dog-IgE. B - Two-dimensional diagram from dog Der $p$ allergogram in Figure $3 \mathrm{~A}$, with the recognized spots identified from $a$ to $x$

may correspond to our 13.5 or 16.5 kDa spots, both with pl 4.7, probably Der p2. Der f1 immunodominant isoforms for humans, present pl 6.6-6.8, but for dogs only the $23.5 \mathrm{kDa}$ band ( $\mathrm{pl}<4.6$ and 5.7 - spots $a$ and $n$ ), probably Der p1, showed major recognition. Towards Der f2, pl 6.9 and 7.2 bands were the immunodominant isoforms for humans, while for dogs, from the probable Der $\mathrm{p} 2$ bands, only the $13.5 \mathrm{kDa}$ one (pl 4.7 - spot g) showed ma- jor recognition. However, the $13.5 \mathrm{kDa}$ band may also correspond to Der $p$ 5, an allergen close to Der f 5 (13-14 kDa with a pl 5.6 major isoform). Der $f 3$, a band close to $30 \mathrm{kDa}$, with 4 isoforms (pl 4.9, 5.2, 5.3 and 5.9) for humans, which is possibly related to Der $p 3$, may correspond to the close to $28.5 \mathrm{kDa}$ ( $\mathrm{pl} 6.8$ and $>6.9$ - spots s and $u$ ) or to the $32.5 \mathrm{kDa}$ (pl 6.9 - spot $t$ ) bands for dogs. Although, these 28.5 and $32.5 \mathrm{kDa}$ dog IgE-recognized bands may 
also correspond to Der p 7. In fact, the $28-31 \mathrm{kDa}$ band ( $p \mid$ 6.4), also observed by Le Mao et al. [25], could be Der $f 7$ and despite a relevant recognition (90\%) of the $32.5 \mathrm{kDa}$ band in our dog population, the level of each molecular allergen recognition between humans and dogs may diverge significantly [9]. Dogs also recognized a $58 \mathrm{kDa}$ minor band (pl 5.4-6.5), close to the 58-60 kDa described band, with molecular and biological activities related to Der $p 4$ [25]. To proceed with the identification and characterization of $D$. pteronyssinus allergome for dogs, the selected population is being increased, several mite recombinant allergens with possible relevance for dogs, are being tested by inhibition assays and specific antibodies to molecular allergens will also be assessed.

As reported by Nuttall et al. [14], several high molecular weight allergens were also recognized by our mite-sensitized atopic population, with the 210-220 kDa band, probably the heavy chains of mite myosin, a minor allergen for humans [23], standing as the most recognized mite allergen in our dog-sensitized population. Similarly to what happens for humans, with Der $f$ allergens showing wide recognition through the high molecular weight (80-83 and 95-101 kDa) acidic range [25], important recognition was also observed for dogs through 110 and $210-220 \mathrm{kDa}$, at the acidic range (Figure $3 \mathrm{~B}$-spots $e, f$ and $o$ ). Those results closely follow Martins et al. [23] data for human Der p IgE-reactivity, where an intense recognition was also observed, with 9 IgE-reactive bands through the pl 5.5-6.1 range.

The determination of individual spectrotypes, mainly 2-D allergograms, has already shown great utility in the identification of implicated allergen molecules in human latex allergy, as demonstrated by Chardin and Peltre [12]. The interpretation of molecular allergen recognition may also be of great importance for an efficient immunotherapy approach [13]. In the veterinary field, demonstration of relevant cross-reaction between Der $p$ and Der $f$ by Masuda et al. [9] perfectly matched the observations from Raffan et al. [16] that dog sensitization to Der $f$ should be mainly due to cross-sensitization with Der $p$, which is more prevalent in the environment and also the primary sensitizing agent. These important observations suggest the interest of pursuing the identification of different mite species allergomes for dogs, which may be of considerable value for veterinary molecular epidemiology of allergy, by allowing rather specific and more effective immunotherapy. This is further exemplified by the observations of Willemse et al. [3], showing no difference in clinical efficiency between immunotherapy with Der $f$ extract or with a placebo, probably because the verified sensitization to Der f resulted mostly from cross reaction with other primary sensitizing mite species, as reported by Raffan et al. [16]. In this case, a possible explanation could be that several sensitizing and clinically important allergens from other mite species, which were not cross-reactive with Der f, would stay out of the immuno- therapy effect of the Der f extract, resulting in no clinical benefit. Resch et al. [26] emphasized the importance of less frequent recognized allergens in humans, like Der $p$ 10, the mite tropomyosin, when allergen-specific immunotherapy is considered, since patients were found suffering from clinically relevant house-dust-mite allergy, due to sensitization to Der p 10.

The usefulness of molecular epidemiology had also been defined by Bessot and Pauli [4], concerning mite allergy in humans, because it would lead to a better understanding of the cross reaction phenomena between different mite species, either from the same family or not. Further knowledge on this subject will probably open new perspectives regarding more specific immunotherapy, based on genetically hypoallergenic variants of major allergens [4].

In our study, after a 1-year course of mite-specific immunotherapy, patient no. 2 showed a remarkable clinical improvement, but increased D. pteronyssinus spectrotype complexity, which could be associated with several Th2like immunological mechanisms, occurred in the initial phase of immunotherapy [27-29], suggesting no absolute correlation between sensitization and allergy. In recent studies carried out by our group, these observations were also made with other allergic patients, regarding different grass pollen spectrotypes, but so far it has not been possible to detect any correlation between those and the efficacy of immunotherapy (unpublished results). More patients should be studied to confirm any possible early/late evolution in sensitization during the course of specific immunotherapy.

Thereafter, deeper characterization of patients, especially in terms of sensitization to other mite species, with the identification of their allergomes and cross-reactive behavior by the inhibitionof ELISA and immunoblottings, will be needed, as it has been proven $[9,16]$. The identification of mite molecular allergens for dogs, by specific antibodies, will also be crucial for a precise identification of the most relevant molecular allergens, allowing the necessary characterization of house-dust mite sensitization and allergy, regarding a more successful immunotherapy.

As a future trend we think that the concept of CRD [18] and consequent CRIT [19] may also be applied to veterinary allergology. In fact, CRD is already being applied to human allergy management through the Immune Solid-phase Allergen Chip (ISAC - Thermo Fisher Scientific, USA) diagnostic facility. However, while these trade mark method is still not available for veterinary purpose, we consider that the identification and characterization of the most relevant allergens, especially for dogs, should continue through other available methods. For a better characterization of these identified allergens, further studies using several allergen-specific antibodies, recombinant allergens and mass spectrometry techniques are presently in the course. 
As for humans, studies of molecular epidemiology in dog allergy will allow clarifying the evolution of allergic response during immunotherapy. Hence, besides the very close clinical patterns between different patients, the individual pattern of sensitization for each allergenic source may also become of an outstanding relevance for the success of a real allergen-specific immunotherapy.

\section{Acknowledgments}

The authors thank BIAL Aristegui (Bilbao, Spain) for kindly providing a part of the intradermal tests; Companhia Portuguesa Consumer Health (CPCH) (Alcabideche, Portugal) for kindly providing several dermatological medications; UNIVET laboratories (Barcelona, Spain) for supporting part of the specific IgE determinations and Sophie Luísa Fechner for proof-reading the text.

This work was conducted in Veterinary Hospital, University of Évora and should be attributed to Department of Veterinary Medicine, School of Sciences and Technology, University of Évora; Institute of Mediterranean Agricultural and Environmental Science (ICAAM), University of Évora.

The work was funded by 1) FEDER Funds through the Operational Program for Competitiveness Factors COMPETE and National Funds through FCT - Foundation for Science and Technology under the Strategic Project PEst-C/AGR/UI0115/2011; 2) SFRH/BPD/48164/2008 andUE/ICAAM/BII/03/2009, Fundação para a Cięncia e Tecnologia, Fundo Europeu de Desenvolvimento Regional, Quadro de Referęncia Estratégico Nacional.

\section{Conflict of interest}

The authors declare no conflict of interest. The sponsors were not involved in the study design, collection, analysis and interpretation of data, in the writing of the manuscript or in the decision to submit it for publication.

\section{References}

1. MacDonald JM. Allergen specific immunotherapy for atopy. In: Proceedings of North American Veterinary Conference 2009. www.ivis.org/proceedings/NAVC/2006/SAE/139.asp?$L A=1$. Accessed Jun 11, 2011.

2. Olivry T, DeBoer DJ, Favrot C, et al. Treatment of canine atopic dermatitis: 2010 clinical practice guidelines from the International Task Force on Canine Atopic Dermatitis. Vet Dermatol 2010; 21: 233-48.

3. Willemse T, Bardagi M, Carlotti DN, et al. Dermatophagoides farinae-specific immunotherapy in atopic dogs with hypersensitivity to multiple allergens: a randomised, double blind, placebo-controlled study. Vet J 2009; 180: 337-42.

4. Bessot JC, Pauli G. Mite allergens: an overview. Eur Ann Allergy Clin Immunol 2011; 43: 141-56.

5. Asturias JA, Ibarrola I, Arilla MC, et al. Engineering of major house dust mite allergens Der $p 1$ and Der $p 2$ for allergen-specific immunotherapy. Clin Exp Allergy 2009; 39: 1088-98.
6. Casquete-Román E, Rosado-Gil T, Postigo I, et al. Contribution of molecular diagnosis of allergy to the management of pediatric patients with allergy to pollen. J Investig Allergol Clin Immunol 2009; 19: 439-45.

7. Hejl C, Wurtzen PA, Kleine-Tebbe J, et al. Phleum pratense alone is sufficient for allergen-specific immunotherapy against allergy to Pooideae grass pollens. Clin Exp Allergy 2009; 39: 752-9.

8. Martínez-Cócera C, Sastre J, Cimarra M, et al. Immunotherapy with a Phleum pratense allergen extract induces an immune response to a grass-mix allergen extract. J Investig Allergol Clin Immunol 2010; 20: 13-9.

9. Masuda K, Tsujimoto H, Fujiwara S, et al. IgE sensitivity and cross-reactivity to crude and purified mite allergens (Der $f 1$, Der f 2, Der p 1, Der p 2) in atopic dogs sensitive to Dermatophagoides mite allergens. Vet Immunol Immunopathol 1999; 72: 303-13.

10. Kubota S, Miyaji K, Shimo Y, et al. IgE reactivity to a Cry j 3 , an allergen of Japanese cedar (Cryptomeria japonica) pollen in dogs with canine atopic dermatitis. Vet Immunol Immunopathol 2012; 149: 132-5.

11. Ognjenovic J, Milcic-Matic N, Smiljanic K, et al. Immunoproteomic characterization of Ambrosia artemisiifolia pollen allergens in canine atopic dermatitis. Vet Immunol Immunopathol 2013; 155: 38-47.

12. Chardin H, Peltre G. Allergome: the characterization of allergens based on a 2D gel electrophoresis approach. Expert Rev Proteomics 2005; 2: 757-65.

13. Pauli G, Malling HJ. The current state of recombinant allergens for immunotherapy. Curr Opin Allergy Clin Immunol 2010; 10: 575-81.

14. Nuttall TJ, Lamb JR, Hill PB. Characterisation of major and minor Dermatophagoides allergens in canine atopic dermatitis. Res Vet Sci 2001; 71: 51-7.

15. McCall C, Hunter S, Stedman K, et al. Characterization and cloning of a major high molecular weight house dust mite allergen (Der f 15) for dogs. Vet Immunol Immunopathol 2001; 78: 231-47.

16. Raffan E, Lawrence $H$, Henderson $T$, et al. Prevalence of the group 1 Dermatophagoides allergens Der $\mathrm{p} 1$ and Der $\mathrm{f} 1$ in homes with no dogs, healthy dogs and Dermatophagoides-sensitized atopic dogs in Liverpool. Vet Dermatol 2001; 16: 253-60.

17. Fall BI, Niessner R. Detection of known allergen-specific IgE antibodies by immunological methods. Methods Mol Biol 2009; 509: 107-22.

18. Jahn-Schmid B, Harwaneggw C, Hillerw R, et al. Allergen microarray: comparison of microarray using recombinant allergens with conventional diagnostic methods to detect allergen-specific serum immunoglobulin E. Clin Exp Allergy 2003; 33: 1443-9.

19. Valenta R, Lidholm J, Niederberger $\mathrm{V}$, et al. The recombinant allergen-based concept of component-resolved diagnostics and immunotherapy (CRD and CRIT). Clin Exp Allergy 1999; 29: 896-904.

20. Olivry T. International task force of canine atopic dermatitis. New diagnostic criteria for canine atopic dermatitis. Vet Dermatol 2010; 21: 123-6.

21. Lee KW, Blankenship KD, McCurry ZM. Performance characteristics of a monoclonal antibody cocktail-based ELISA for detection of allergen-specific IgE in dogs and comparison with a high affinity IgE receptor-based ELISA. Vet Dermatol 2009; 20: 157-64.

22. Muller H, Schweizer B. Biochemical applications for UV/Vis spectroscopy. Perkin-Elmer Corp. Norwalk, USA 1993. 
23. Martins LM, Peltre G, Faro C, et al. The Helix aspersa (Brown Garden Snail) allergen repertoire. Int Arch Allergy Immunol 2005; 136: 7-15.

24. Westermeier R. Electrophoresis in practice. VCH Germany 1993.

25. Le Mao J, Mayer CE, Peltre G, et al. Mapping of Dermatophagoides farinae mite allergens by two-dimensional immunoblotting. J Allergy Clin Immunol 1998; 102: 631-6.

26. Resch Y, Weghofer M, Seiberler S, et al. Molecular characterization of Der p 10: a diagnostic marker for broad sensitization in house dust mite allergy. Clin Exp Allergy 2011; 41: 1468-77.

27. Kindt TJ. Hypersensitivity reactions. In: Kuby immunology. Kindt TJ, Goldsby RA, Osborne BA (eds.) 6th ed. W.H. Freeman and Company. USA 2007; 371-400.

28. Jutel M, Akdis M, Budak F, et al. IL-10 and TGF-beta cooperate in the regulatory $T$ cell response to mucosal allergens in normal immunity and specific immunotherapy. Eur J Immunol 2003; 33: 1205-14.

29. Creticos PS, Van Metre TE, Mardiney MR, et al. Dose response of IgE and IgG antibodies during ragweed immunotherapy. J Allergy Clin Immunol 1984; 73: 94-104. 